\title{
Evaluation of Contrast Sensitivity, Chromatic Vision, and Reading Ability in Patients with Primary Open Angle Glaucoma
}

\author{
Maria P. Bambo, ${ }^{1,2}$ Blanca Ferrandez, ${ }^{1,2}$ Noemi Güierri, ${ }^{1,2}$ Isabel Fuertes, ${ }^{1,2}$ \\ Beatriz Cameo, ${ }^{1,2}$ Vicente Polo, $^{1,2}$ Jose M. Larrosa, ${ }^{1,2}$ and Elena Garcia-Martin ${ }^{1,2}$ \\ ${ }^{1}$ Miguel Servet University Hospital, Ophthalmology Department, Zaragoza, Spain \\ ${ }^{2}$ Aragon Institute for Health Research (IIS Aragón), University of Zaragoza, Zaragoza, Spain
}

Correspondence should be addressed to Maria P. Bambo; mpbamborubio@gmail.com

Received 28 September 2016; Accepted 11 October 2016

Academic Editor: Van C. Lansingh

Copyright (C) 2016 Maria P. Bambo et al. This is an open access article distributed under the Creative Commons Attribution License, which permits unrestricted use, distribution, and reproduction in any medium, provided the original work is properly cited.

Purpose. To compare contrast sensitivity, acquired color vision deficiency, and reading ability in patients with glaucoma at different stages of the disease and to establish correlations between visual field parameters and visual function scores. Methods. This prospective cross-sectional study included 121 glaucoma patients. Subjects with a diagnosis of chronic open angle glaucoma were recruited and classified according to Hodapp-Parrish-Anderson criteria. Patients with severe visual field defects were excluded because they were older, which could bias the interpretation of visual function tests. Contrast sensitivity was measured using the Pelli-Robson Chart and the CSV1000E test. Chromatic vision was evaluated using the Farnsworth-panel D15 and the L'Anthony D15 tests of Vision Color Recorder software. Reading ability was measured using Radner-Vissum test. Results. Contrast sensitivity (with photopic and mesopic luminance with glare) differed significantly between patients with early and moderate visual field defects $(p<0.05)$. Reading ability scores and results of the chromatic vision tests did not differ significantly between the two groups. Significant and moderate Spearman correlations between visual field indexes and contrast sensitivity tests were detected. Conclusions. Contrast sensitivity was significantly worse in patients with moderate glaucoma compared to those with early-stage glaucoma. Evaluation of visual function in clinical practice provides important information to address a glaucoma patient's vision complaints.

\section{Introduction}

Vision loss due to glaucoma is traditionally described as a loss of "peripheral vision" [1], although this is not the most commonly reported symptom. Requiring more light and blurry vision are the most common complaints of patients with glaucoma [2].

Even from early stages of the disease, glaucoma patients often complain of much worse vision than we would expect based on their good visual acuity; and this apparent discrepancy may be based on the decrease in visual function caused by glaucoma. Visual ability in low illumination conditions and the capability to detect low-contrast objects are two important functions in daily life of patients with peripheral vision loss due to glaucoma [3]. Recent studies suggest that glaucoma is also associated with greater self-reported reading difficulty [4] and modestly lower reading speeds [5]. Color vision defects in patients with glaucoma, especially along the blue-yellow axis, are also reported [6].

The goal of this study was to evaluate a large sample of glaucoma patients at different stages of disease severity to further investigate contrast sensitivity, color vision, and reading ability using various psychophysical tests. To the best of our knowledge, there are no studies that evaluate contrast sensitivity in glaucoma patients under different lighting conditions (photopic and mesopic with glare condition, which could resemble night driving). In addition, we evaluated the correlation between visual function worsening and visual field damage in glaucoma. Characterizing visual function in patients with glaucoma (beyond the visual field) may 
TABLE 1: Hodapp-Parrish-Anderson classification system.

\begin{tabular}{|c|c|c|}
\hline Early defect & Moderate defect & Severe defect \\
\hline $\begin{array}{l}\text { Mean deviation between } 0 \text { and }-6 \mathrm{~dB} \text {, AND } \\
\text { at least one of the following listed criteria: }\end{array}$ & $\begin{array}{l}\text { Mean deviation between }-6 \text { and }-12 \mathrm{~dB} \text {, } \\
\text { AND at least one of the following listed } \\
\text { criteria: }\end{array}$ & $\begin{array}{l}\text { Mean deviation }<-12 \mathrm{~dB}, \mathrm{AND} \text { at least one } \\
\text { of the following listed criteria: }\end{array}$ \\
\hline $\begin{array}{l}\text { (i) A cluster of } \geq 3 \text { points on the pattern } \\
\text { deviation plot in an expected location of the } \\
\text { visual field depressed below the } 5 \% \text { level, at } \\
\text { least one of which is depressed below the } 1 \% \\
\text { level, OR } \\
\text { (ii) Pattern standard deviation significant at } \\
p<0.05 \text {, OR } \\
\text { (iii) Glaucoma Hemifield Test = Outside } \\
\text { Normal Limits. }\end{array}$ & $\begin{array}{l}\text { (i) } \geq 25 \% \text { but }<50 \% \text { of points on the pattern } \\
\text { deviation plot depressed below the } 5 \% \text { level, } \\
\text { and } \geq 15 \% \text { but }<25 \% \text { of points depressed } \\
\text { below the } 1 \% \text { level, OR } \\
\text { (ii) At least } 1 \text { point within the central } 5^{\circ} \text { with } \\
\text { sensitivity of }<15 \mathrm{~dB} \text {, but no points in the } \\
\text { central } 5^{\circ} \text { can have a sensitivity of } 0 \mathrm{~dB} \text {, OR } \\
\text { (iii) Only one hemifield may have a point } \\
\text { with sensitivity of }<15 \mathrm{~dB} \text { within } 5^{\circ} \text { of } \\
\text { fixation. }\end{array}$ & $\begin{array}{l}\text { (i) } \geq 50 \% \text { but }<75 \% \text { of points on pattern } \\
\text { deviation plot depressed below the } 5 \% \text { level } \\
\text { and } \geq 25 \% \text { but }<50 \% \text { of points depressed } \\
\text { below the } 1 \% \text { level, OR } \\
\text { (ii) At least one point in the central } 5^{\circ} \text { has a } \\
\text { sensitivity of } 0 \mathrm{~dB} \text {, OR } \\
\text { (iii) Points within the central } 5^{\circ} \text { with } \\
\text { sensitivity }<15 \mathrm{~dB} \text { in both hemifields. }\end{array}$ \\
\hline
\end{tabular}

dB: decibels.

be useful toward enhancing our knowledge and developing potential methods for enhancing vision-related function in these patients.

\section{Materials and Methods}

The local ethics committee approved the study procedure, which was conducted in accordance with the Declaration of Helsinki. Informed consent was obtained from all participants.

2.1. Study Subjects. All glaucoma patients were recruited from the Glaucoma Department at the Miguel Servet University Hospital (Zaragoza, Spain).

Inclusion criteria included a clinical diagnosis of glaucoma at a previous visit at least 1 year before. Patients with primary open angle glaucoma, normal-tension glaucoma, pseudoexfoliative glaucoma, or pigment dispersion glaucoma were included. A diagnosis of glaucoma was based on characteristic optic nerve damage on slit-lamp examination (defined as a definite notch in the neuroretinal rim or absence of the neuroretinal rim not due to another known cause) with corresponding visual field defects. Only eyes with best corrected visual acuity (BCVA) of 20/30 or better based on the Snellen chart were included.

Subjects were excluded if they had vision loss secondary to another eye condition, had any laser procedure in the previous month, or had any ocular surgery in the previous 3 months. Other exclusion criteria included extreme refractive errors such as high myopia ( -5.0 or higher), hyperopia $(+5.0$ or higher), or astigmatism $( \pm 3.0$ or higher) or acute angle closure glaucoma, congenital color vision defects, or history of stroke or neurologic pathology. Patients with clinically significant lenticular opacity using the LOCS III classification [7] were also excluded. The exclusion criteria for lenticular opacity were nuclear color/opalescence greater than NC2 and NO2, respectively, cortical cataract greater than $\mathrm{C} 2$, and posterior subcapsular cataract greater than or equal to P1.
2.2. Ophthalmological Examination. The ocular examination included measurements of BCVA using ETDRS Charts (Precision Vision, IL, USA) at $4 \mathrm{~m}$ with decimal Snellen visual acuity notation and intraocular pressure (IOP) (using a calibrated Goldmann applanation tonometer), slit-lamp examination of the anterior segment, and fundus evaluation. Given the influence of pupil size in psychophysical testing [8], horizontal pupil diameter measurements were obtained using a Colvard pupillometer (Oasis Medical, Glendora, California) in photopic lighting at 120 candelas $/ \mathrm{m}^{2}\left(\mathrm{~cd} / \mathrm{m}^{2}\right)$. The Humphrey 24-2 Swedish Interactive Threshold Algorithm Standard perimeter (Zeiss Meditec, Dublin, CA) was used to evaluate the visual field. All enrolled subjects were classified according to two stages of visual field damage using the Hodapp-Parrish-Anderson criteria [9]: early and moderate defects (Table 1). Patients with severe defects were excluded because they were older, which could bias the interpretation of visual function tests.

2.3. Contrast Sensitivity Evaluation. The Pelli-Robson chart test and the CSV-1000E were used to evaluate contrast sensitivity. The Pelli-Robson test is a contrast sensitivity test with 8 lines and 16 levels of horizontal capital letters measuring central vision. Each set of three letters on the chart becomes progressively lower in contrast relative to the background. Each eye is evaluated separately with the nontested eye occluded. The chart is mounted on a white wall with the patient sitting 1 meter away from the chart; the luminance of the test is photopic, at $120 \mathrm{~cd} / \mathrm{m}^{2}$, with the accepted range being between 60 and $120 \mathrm{~cd} / \mathrm{m}^{2}$ [10].

The CSV-1000E chart (Vector Vision, Haag-Streit, Harlow, UK) is a contrast sensitivity chart test with four rows of sine-wave gratings. At the recommended test distance of $2.5 \mathrm{~m}$, these gratings test the spatial frequencies of $3,6,12$, and 18 cycles/degree (cpd) to establish a functional acuity score for patients under nonglare and glare conditions [11]. Each eye is evaluated separately with the nontested eye occluded. A functional acuity score can be obtained directly from the testscoring sheet, and log values were selected. Ambient lighting 
used to conduct the test was photopic $\left(120 \mathrm{~cd} / \mathrm{m}^{2}\right)$ without glare and mesopic $\left(0.8 \mathrm{~cd} / \mathrm{m}^{2}\right)$ with glare condition (after $10 \mathrm{~min}$ of dark adaptation, at $0.8 \mathrm{~cd} / \mathrm{m}^{2}$ ).

2.4. Color Vision Evaluation. The Farnsworth-panel D15 (FD15) and the L'Anthony desaturated D15 (L-D15) tests of Vision Color Recorder software (Optical Diagnosis, Inc., Beusichem, Netherlands) were performed in all the study subjects using the best corrected refraction for near sight under specific photopic lighting $\left(120 \mathrm{~cd} / \mathrm{m}^{2}\right)$ and at a $45^{\circ}$ angle to the screen. Each eye was evaluated separately with the nontested eye occluded. F-D15 and L-D15 are both arrangement tests in which the subject is offered a series of colors that need to be sorted either into a sequence (usually based on hue) or into groups (most often grays versus colors). The L-D15 test is more sensitive than the F-D15 test for detecting color vision impairment. Therefore, a subject who passes the F-D15 test but fails the L-D15, which has smaller color differences, may have a mild defect in chromatic vision.

For grading acquired color vision defects, we used the Confusion Color Index (CCI) developed by Bowman [12]. CCI assesses the severity of dyschromatopsia. A CCI score higher than 1 indicates altered color vision perception; the higher the score, the worse the condition.

2.5. Reading Ability Evaluation. Radner et al. developed the Radner reading charts, which are based on the concept of using sentence optotypes for the standardized examination of reading acuity and reading speed [13]. The Spanish version of the Radner reading charts, Radner-Vissum test, was administered to all of the study patients binocularly [14]. The reading distance was $40 \mathrm{~cm}$. A standard luminance of $80 \mathrm{~cd} / \mathrm{m}^{2}$ was used.

The parameters registered were $\operatorname{LogRAD}$ score and maximum reading speed (MRS) in words per minute (wpm). $\operatorname{LogRAD}$ score is a correction of the reading acuity $(\operatorname{LogRAD})$ for the number of reading errors (LogRAD score $=\operatorname{LogRAD}$ $+0.005 \times$ syllables of incorrectly read words). The reading speed was calculated based on the number of words in a sentence and the time needed to read the sentence (14 words $\times$ 60 seconds divided by the reading time $=$ words per minute) . The mean reading speed of the subsequent sentences up to the Critical Print Size was defined as the MRS. The Critical Print Size is the print size at which reading speed begins to deteriorate; after calculating the reading speed per sentence (wpm), this was plotted on a graph and the print size where the line suddenly dropped was defined as the Critical Print Size.

2.6. Statistical Analysis. This was an observational, prospective cross-sectional study. A total of 121 eyes of 121 patients were included in the statistical analysis. One eye from each subject was randomly chosen for the study, unless only one eye met the inclusion criteria. All 121 eyes were stratified according to the severity of glaucomatous damage in two stages (early and moderate defects) following HodappParrish-Anderson criteria [9] (Table 1). All data analyses were performed using SPSS version 20.0 (SPSS Incorporation,
TABle 2: Descriptive and clinical data for 121 glaucoma patients classified into two groups according to disease severity (HodappParrish-Anderson criteria).

\begin{tabular}{lccc}
\hline & $\begin{array}{c}\text { Early defect } \\
(n=94) \\
\text { Mean } \pm \text { SD }\end{array}$ & $\begin{array}{c}\text { Moderate defect } \\
(n=27)\end{array}$ & ${ }^{*} p$ \\
& Mean \pm SD & \\
\hline Age (years) & $65.13 \pm 9.37$ & $66.44 \pm 10.03$ & 0.253 \\
$\begin{array}{l}\text { BCVA (decimal } \\
\text { notation) }\end{array}$ & $1.07 \pm 0.23$ & $0.94 \pm 0.20$ & $\mathbf{0 . 0 1 6}$ \\
IOPb (mm Hg) & $22.03 \pm 2.86$ & $22.37 \pm 3.80$ & 0.647 \\
CDR & $0.58 \pm 0.19$ & $0.75 \pm 0.17$ & $<\mathbf{0 . 0 0 1}$ \\
MD (dB) & $-1.95 \pm 1.42$ & $-8.74 \pm 1.79$ & $<\mathbf{0 . 0 0 1}$ \\
PSD (dB) & $2.19 \pm 1.31$ & $8.98 \pm 2.49$ & $<\mathbf{0 . 0 0 1}$ \\
VFI (\%) & $97.23 \pm 3.02$ & $78.08 \pm 6.22$ & $<\mathbf{0 . 0 0 1}$ \\
CP ( $\mu$ m) & $552.72 \pm 41.80$ & $545.92 \pm 32.49$ & 0.239 \\
Photopic pupil size & $3.29 \pm 0.78$ & $3.27 \pm 0.63$ & 0.803 \\
(mm) & & & \\
\hline
\end{tabular}

${ }^{*} p$ : level of statistical significance in comparison between groups using the Mann-Whitney nonparametric test. Bold text shows the statistically significant results $(p<0.05)$.

BCVA: best corrected visual acuity; IOPb: basal intraocular pressure; CDR: cup-to-disc ratio; MD, PSD, and VFI, mean deviation (MD), pattern standard deviation (PSD), and visual field index (VFI) obtained in the visual field using Humphrey 24-2 Swedish Interactive Threshold Algorithm Standard perimeter; dB: decibels; $\mathrm{CP}$ : corneal pachymetry; $\mu \mathrm{m}$ : micrometers; $\mathrm{SD}$ : standard deviation.

Chicago, IL) statistical software. The Kolmogorov-Smirnov test was used to assess sample distribution. Due to the nonparametric distribution of the data, the results were compared using the Mann-Whitney nonparametric test. Correlations were examined by Spearman's test. A $p$ value of 0.05 was considered statistically significant.

\section{Results}

One hundred and twenty-one eyes of 121 glaucoma patients were examined (94 eyes with early defects and 27 with moderate defects). Epidemiologic and disease characteristics of each of the two groups of patients are shown in Table 2. Sixty-four patients were females (52.9\%) and 57 were males (47.1\%). The two groups did not differ with respect to age or sex. Pupil size did not differ between the groups.

The visual function results are summarized in Table 3 . The contrast sensitivity evaluation indicated statistical differences of the Pelli-Robson and CSV1000E parameters (photopic luminance; all frequencies) between patients with early and moderate defects $(p<0.05)$. The CSV1000E test performed with mesopic luminance and glare conditions also revealed statistically significant differences in all frequencies except 12 cpd between patients with early and moderate defects $(p<$ $0.05)$. Our analysis revealed no significant differences in color vision and reading ability between the early and moderate defect groups.

Correlations between visual field indexes and contrast sensitivity tests were moderate and statistically significant 
TABLE 3: Mean and standard deviation obtained in visual function tests with subjects classified into two groups according to disease severity (Hodapp-Parrish-Anderson criteria).

\begin{tabular}{lccc}
\hline & $\begin{array}{c}\text { Early defect } \\
(n=94)\end{array}$ & $\begin{array}{c}\text { Moderate } \\
\text { defect } \\
(n=27)\end{array}$ & $p^{*}$ \\
& Mean \pm SD & Mean \pm SD & \\
\hline Pelli-Robson (F) & $1.76 \pm 0.18$ & $1.51 \pm 0.30$ & $<\mathbf{0 . 0 0 1}$ \\
CSV (F) 3 cpd & $1.70 \pm 0.22$ & $1.51 \pm 0.26$ & $\mathbf{0 . 0 0 3}$ \\
CSV (F) 6 cpd & $1.87 \pm 0.24$ & $1.68 \pm 0.29$ & $\mathbf{0 . 0 0 3}$ \\
CSV (F) 12 cpd & $1.45 \pm 0.30$ & $1.24 \pm 0.37$ & $\mathbf{0 . 0 2 2}$ \\
CSV (F) 18 cpd & $1.02 \pm 0.32$ & $0.73 \pm 0.35$ & $\mathbf{0 . 0 0 2}$ \\
CSV (M + G) 3 cpd & $1.48 \pm 0.31$ & $1.25 \pm 0.34$ & $\mathbf{0 . 0 0 1}$ \\
CSV (M + G) 6 cpd & $1.29 \pm 0.56$ & $0.88 \pm 0.61$ & $\mathbf{0 . 0 0 3}$ \\
CSV (M + G) 12 cpd & $0.73 \pm 0.56$ & $0.46 \pm 0.42$ & 0.062 \\
CSV (M + G) 18 cpd & $0.40 \pm 0.39$ & $0.18 \pm 0.21$ & $\mathbf{0 . 0 2 0}$ \\
LogRad score (RV) & $0.09 \pm 0.10$ & $0.11 \pm 0.15$ & 0.668 \\
MRS (wpm) & $195.6 \pm 36.1$ & $189.4 \pm 62.1$ & 0.110 \\
CCI F-D15 & $1.16 \pm 0.24$ & $1.12 \pm 0.20$ & 0.733 \\
CCI L-D15 & $1.36 \pm 0.33$ & $1.43 \pm 0.27$ & 0.173 \\
\hline
\end{tabular}

* $p$ : level of statistical significance in comparison between groups using Mann-Whitney nonparametric test. Bold text shows the statistically significant results $(p<0.05)$.

RV: Radner-Vissum reading test; MRS: maximum reading speed; wpm: words per minute; F: photopic luminance; CSV: CSV1000E contrast sensitivity test; $\mathrm{M}+\mathrm{G}$ : mesopic luminance with glare condition; cpd: cycles per degree (spatial frequency); CCI: confusion color index; F-D15: Farnsworthpanel D15; L-D15: L'Anthony desaturated D15; SD: standard deviation.

(Table 4). The highest correlations were between the PelliRobson results and mean deviation $(r=0.43, p<0.001)$; Pelli-Robson and pattern standard deviation $(r=-0.48$, $p<0.001)$; and Pelli-Robson and visual field index $(r=$ $0.38, p<0.001)$. Correlations were lower ( $r$ absolute value ranged between 0.25 and 0.39 ), but statistically significant, between almost all variables of the visual field indexes (especially mean deviation) and CSV1000E results performed in photopic luminance and mesopic luminance with glare, with the best correlations in the low frequencies (3 and 6 cpd). Visual field indexes and reading ability results were not significantly correlated. The visual field indexes and CCI were not significantly correlated, except for pattern standard deviation and CCI of L-D15 with a significant weak correlation $(r=0.21, p=0.019)$.

\section{Discussion}

Although visual field measurement and optic disc evaluation are the principal methods for confirming the presence and progression of glaucoma, psychophysical tests to examine the function of specific parts of the visual pathway are also useful for monitoring glaucomatous changes. The decline in spatial contrast sensitivity in patients with glaucoma has been documented in different tests [15]. The Pelli-Robson chart produces reliable and reproducible results [16]. The Pelli-Robson chart tests the spatial frequency of $1 \mathrm{cpd}$ at a distance of 1 meter. Patients with ocular hypertension and glaucoma demonstrate contrast sensitivity losses at spatial frequencies between 0.25 and $8 \mathrm{cpd}$ [17]. Our findings indicated that contrast sensitivity was worse in glaucoma patients with moderate defects than in patients with early defects, especially at low frequencies $(<6 \mathrm{cpd})$, for both photopic and mesopic luminance with glare lighting. There was a moderate correlation between worsening visual field indexes and decreased contrast sensitivity, stronger with the Pelli-Robson than the CSV1000E test. Our result is consistent with other findings, such as those by Hawkins et al. [18] and Wilensky et al. [19], who reported a significant correlation between the mean deviation as measured with the Humphrey perimeter and the Pelli-Robson contrast sensitivity scores $(r=0.56, p<0.001, n=127 ; r=0.59, p<0.001, n=120$, resp.). Others report that contrast sensitivity can be more affected than high-contrast visual acuity in glaucoma patients $[20,21]$. Although previous studies did not provide enough evidence to support the use of contrast sensitivity for the early detection of glaucoma [22, 23], contrast sensitivity correlates with the perimeter deviation $[20,21]$ and we believe that contrast sensitivity in conjunction with visual field testing will be a promising method of detecting functional changes in glaucoma patients-even in those with good visual acuity.

Regarding color vision examination, CCI obtained in the F-D15 and L-D15 tests did not differ among groups. Another study in patients with primary open angle glaucoma reported a prevalence of $60 \%$ for blue-yellow defects in contrast to only $3 \%$ for red-green defects [24]. Several theories have been introduced to explain tritan-like defects in glaucoma, such as a greater susceptibility of blue-yellow sensitive ganglion cells to IOP-related damage due to their morphology and connectivity to second-order neurons [25]. Our results suggest that patients with glaucoma manifest signs of deteriorating color discrimination ability (CCI $>1$ in both groups), but we did not detect differences in color discrimination according to disease severity or a significant correlation between the results of color perception testing and visual field indexes. Moreover, age could have a potentially strong effect on the deterioration of CCI, as suggested in Vingrys and King-Smith [26].

Differences in reading ability were also not detected between groups based on severity in our study, and we detected no significant correlation with visual field indexes and reading scores. Several studies have demonstrated reading impairments in subjects with glaucoma through performance-based reading testing different from the Radner-Vissum test $[5,27]$. Performance-based reading tests are commonly measured in strictly controlled conditions, which do not reflect the typical daily environment of the patient, and this kind of test could be subject to wide variability. Glaucoma patients often complain about problems with reading, especially small print, but the reading impairment reported in glaucoma patients [28] may be due to a number of mechanisms, including poor detection of low-contrast stimuli or inadequate lighting conditions, and this must be taken into account in clinical practice to suggest methods to enhance reading ability (creating proper lighting to optimize contrast or teaching strategies to mitigate 
TABLE 4: Spearman correlation coefficients $(r)$ between visual field parameters and contrast sensitivity, reading ability, and color vision measures.

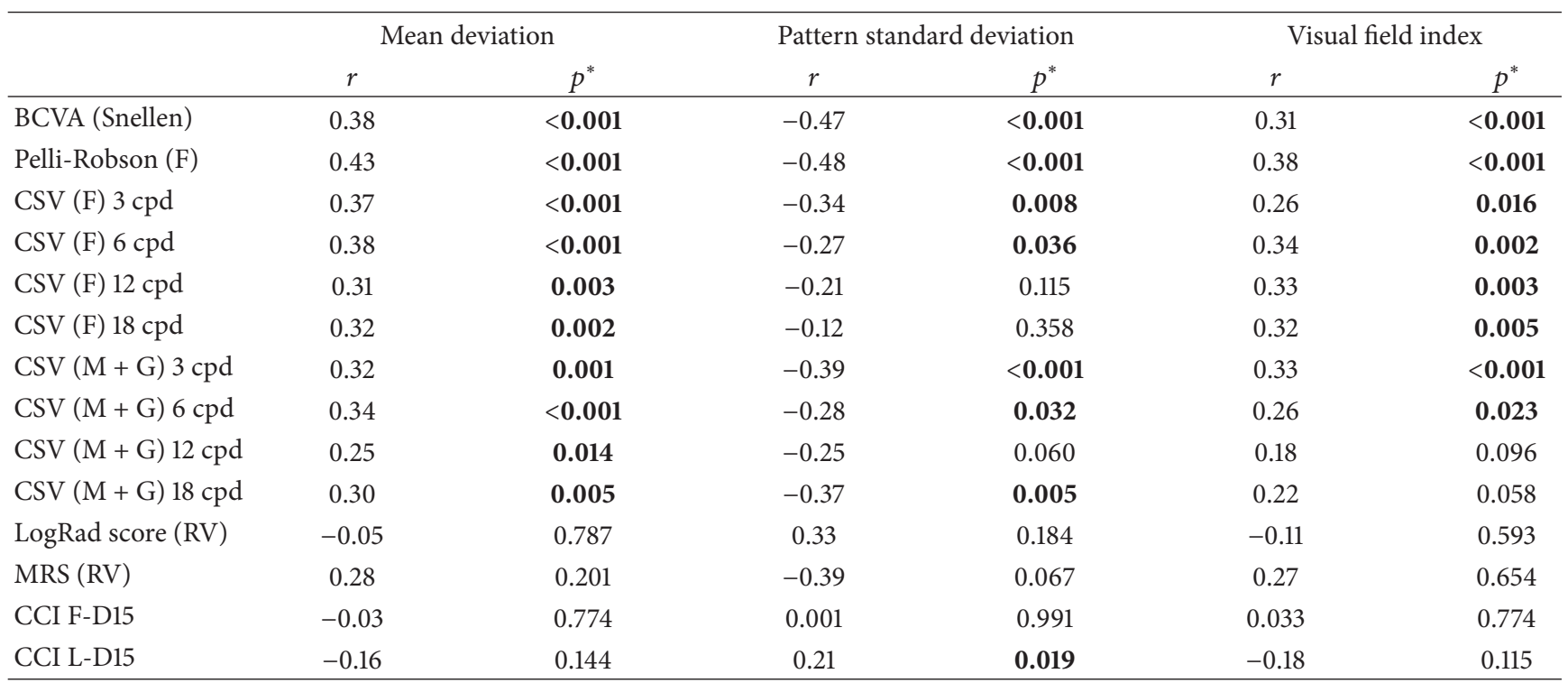

${ }^{*} p$ : level of statistical significance in Spearman correlations. Bold text shows the statistically significant results $(p<0.05)$.

BCVA: best corrected visual acuity; F: photopic luminance; CSV: CSV1000E contrast sensitivity test; M + G: mesopic luminance with glare condition; cpd: cycles per degree (spatial frequencies); RV: Radner-Vissum reading test; MRS: maximum reading speed; CCI: confusion color index; F-D15: Farnsworth-panel D15; L-D15: L’Anthony desaturated D15.

fatigue). Considering the fact that in glaucoma visual acuity is preserved until advanced stages, it is expectable that reading ability is not affected so much for a long time. Further studies examining reading ability under dim light conditions and including patients with severe visual field defects are required to clarify this.

A possible limitation of our study is the presence of cataracts. We assume that the influence of cataracts in visual function measurements in this study was small because we excluded patients with BCVA worse than 20/30 or if they had clinically significant lenticular opacity based on the LOCS III classification [7]. Another limitation of the study was the difference in group size, which contributed to the data not conforming to the normal distribution and, consequently, a reduction in the statistical power that decreased the possibility of detecting differences.

The findings of the present study indicate that contrast sensitivity is more decreased as the severity of glaucoma increases, and this could account for the common complaint of patients with glaucoma of hazy or blurry vision despite good visual acuity. Contrast sensitivity was more closely related than color perception or reading ability with disease severity. The ability to distinguish contrast plays an important role in patients' everyday vision, and several tests are available to distinguish contrast [29]. The Pelli-Robson chart test is a low-technology, inexpensive method for measuring spatial contrast sensitivity, which can detect worsening visual function in glaucoma patients. We recommended its application to document worsening visual function in glaucoma patients. Changes in contrast sensitivity are a potential indicator of the level of glaucoma damage; however, prospective studies are required.

\section{Competing Interests}

No conflicting relationship exists for any author.

\section{Acknowledgments}

This study has been supported in part by the Aragon Institute for Health Research (IIS Aragón).

\section{References}

[1] K. Heilmann and K. T. Richardson, Glaucoma: Conceptions of a Disease, WB Saunders, Philadelphia, Pa, USA, 1978.

[2] C. X. Hu, C. Zangalli, M. Hsieh et al., "What do patients with glaucoma see? Visual symptoms reported by patients with glaucoma," American Journal of the Medical Sciences, vol. 348, no. 5, pp. 403-409, 2014.

[3] J. P. Szlyk, D. P. Taglia, J. Paliga, and J. T. Wilensky, “Driving performance in patients with mild to moderate glaucomatous clinical vision changes," Journal of Rehabilitation Research \& Development, vol. 39, no. 4, pp. 467-482, 2002.

[4] C. M. Mangione, S. Berry, K. Spritzer et al., "Identifying the content area for the 51-item National Eye Institute Visual Function Questionnaire: results from focus groups with visually impaired persons," Archives of Ophthalmology, vol. 116, no. 2, pp. 227-233, 1998.

[5] P. Y. Ramulu, B. K. Swenor, J. L. Jefferys, D. S. Friedman, and G. S. Rubin, "Difficulty with out-loud and silent reading in Glaucoma," Investigative Ophthalmology and Visual Science, vol. 54, no. 1, pp. 666-672, 2013.

[6] D. Poinoosawmy, S. Nagasubramanian, and J. Gloster, "Colour vision in patients with chronic simple glaucoma and ocular hypertension," British Journal of Ophthalmology, vol. 64, no. 11, pp. $852-857,1980$. 
[7] L. T. Chylack Jr., J. K. Wolfe, D. M. Singer et al., “The lens opacities classification system III. The Longitudinal Study of Cataract Study Group," Archives of Ophthalmology, vol. 111, no. 6, pp. 831-836, 1993.

[8] N. C. Strang, D. A. Atchison, and R. L. Woods, "Effects of defocus and pupil size on human contrast sensitivity," Ophthalmic and Physiological Optics, vol. 19, no. 5, pp. 415-426, 1999.

[9] E. Hodapp, R. K. Parrish II, and D. R. Anderson, Clinical Decisions in Glaucoma, The CV Mosby Co, St. Louis, Mo, USA, 1993.

[10] M. Mäntyjärvi and T. Laitinen, "Normal values for the PelliRobson contrast sensitivity test," Journal of Cataract and Refractive Surgery, vol. 27, no. 2, pp. 261-266, 2001.

[11] G. N. Pomerance and D. W. Evans, "Test-retest reliability of the CSV-1000 contrast test and its relationship to glaucoma therapy," Investigative Ophthalmology \& Visual Science, vol. 35, no. 9, pp. 3357-3361, 1994.

[12] K. J. Bowman, "A method for quantitative scoring of the Farnsworth Panel D-15," Acta Ophthalmologica, vol. 60, no. 6, pp. 907-916, 1982.

[13] W. Radner, W. Obermayer, S. Richter-Mueksch, U. Willinger, M. Velikay-Parel, and B. Eisenwort, "The validity and reliability of short German sentences for measuring reading speed," Graefe's Archive for Clinical and Experimental Ophthalmology, vol. 240, no. 6, pp. 461-467, 2002.

[14] J. L. Alió, W. Radner, A. B. Plaza-Puche et al., "Design of short Spanish sentences for measuring reading performance: radnerVissum test," Journal of Cataract and Refractive Surgery, vol. 34, no. 4, pp. 638-642, 2008.

[15] S. L. Teoh, D. Allan, G. N. Dutton, and W. S. Foulds, "Brightness discrimination and contrast sensitivity in chronic glaucoma-a clinical study," British Journal of Ophthalmology, vol. 74, no. 4, pp. 215-219, 1990.

[16] D. B. Elliott, K. Sanderson, and A. Conkey, "The reliability of the Pelli-Robson contrast sensitivity chart," Ophthalmic and Physiological Optics, vol. 10, no. 1, pp. 21-24, 1990.

[17] W. H. Seiple, "The clinical utility of spatial contrast sensitivity testing," Duane's Foundations of Clinical Ophthalmology, vol. 2, no. 114, pp. 1-13, 1991.

[18] A. S. Hawkins, J. P. Szlyk, Z. Ardickas, K. R. Alexander, and J. T. Wilensky, "Comparison of contrast sensitivity, visual acuity, and Humphrey visual field testing in patients with glaucoma," Journal of Glaucoma, vol. 12, no. 2, pp. 134-138, 2003.

[19] J. T. Wilensky, A. Hawkins, and R. P. Mills, "Comparison of contrast sensitivity visual acuity, and Humphrey visual field testing in patients with glaucoma," Transactions of the American Ophthalmological Society, vol. 99, pp. 213-218, 2001.

[20] M. Zulauf and J. Flammer, "Correlation of spatial contrast sensitivity and visual fields in glaucoma," Graefe's Archive for Clinical and Experimental Ophthalmology, vol. 231, no. 3, pp. 146-150, 1993.

[21] F. Horn, P. Martus, and M. Korth, "Comparison of temporal and spatiotemporal contrast-sensitivity tests in normal subjects and glaucoma patients," German Journal of Ophthalmology, vol. 4, no. 2, pp. 97-102, 1995.

[22] P. A. Sample, P. S. C. Juang, and R. N. Weinreb, "Isolating the effects of primary open-angle glaucoma on the contrast sensitivity function," American Journal of Ophthalmology, vol. 112, no. 3, pp. 308-316, 1991.

[23] J. M. Wood and J. E. Lovie-Kitchin, "Evaluation of the efficacy of contrast sensitivity measures for the detection of early primary open-angle glaucoma," Optometry and Vision Science, vol. 69, no. 3, pp. 175-181, 1992.

[24] J. Francois and G. Verriest, "Acquired dyschromatopsia in primary glaucoma," Annales d'Oculistique, vol. 192, no. 3, pp. 191-199, 1959.

[25] T. M. Nork, "Acquired color vision loss and a possible mechanism of ganglion cell death in glaucoma," Transactions of the American Ophthalmological Society, vol. 98, pp. 331-363, 2000.

[26] A. J. Vingrys and P. E. King-Smith, "A quantitative scoring technique for panel tests of color vision," Investigative Ophthalmology \& Visual Science, vol. 29, no. 1, pp. 50-63, 1988.

[27] U. Altangerel, G. L. Spaeth, and W. C. Steinmann, "Assessment of function related to vision (AFREV)," Ophthalmic Epidemiology, vol. 13, no. 1, pp. 67-80, 2006.

[28] A. M. Nguyen, S. W. van Landingham, R. W. Massof, G. S. Rubin, and P. Y. Ramulu, "Reading ability and reading engagement in older adults with glaucoma," Investigative Ophthalmology and Visual Science, vol. 55, no. 8, pp. 5284-5290, 2014.

[29] J. Richman, G. L. Spaeth, and B. Wirostko, "Contrast sensitivity basics and a critique of currently available tests," Journal of Cataract \& Refractive Surgery, vol. 39, no. 7, pp. 1100-1106, 2013. 


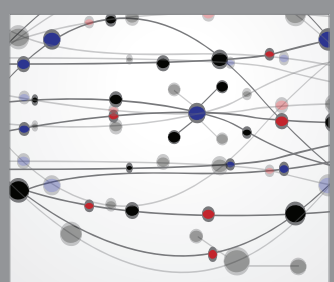

The Scientific World Journal
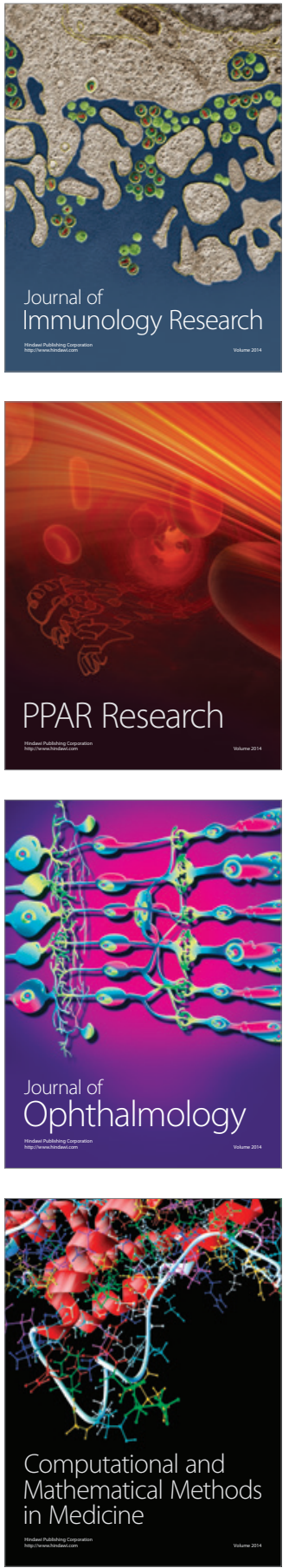

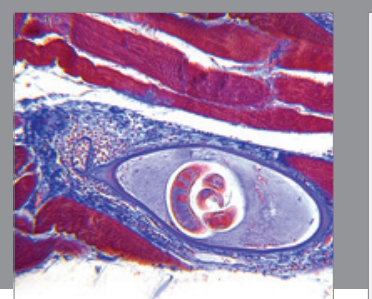

Gastroenterology Research and Practice

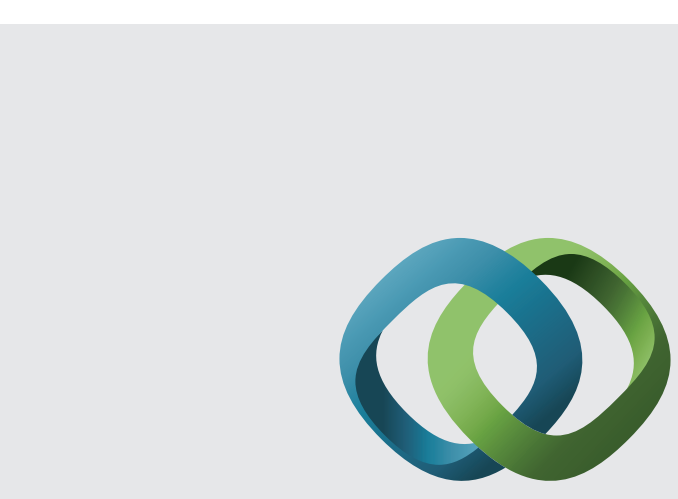

\section{Hindawi}

Submit your manuscripts at

http://www.hindawi.com
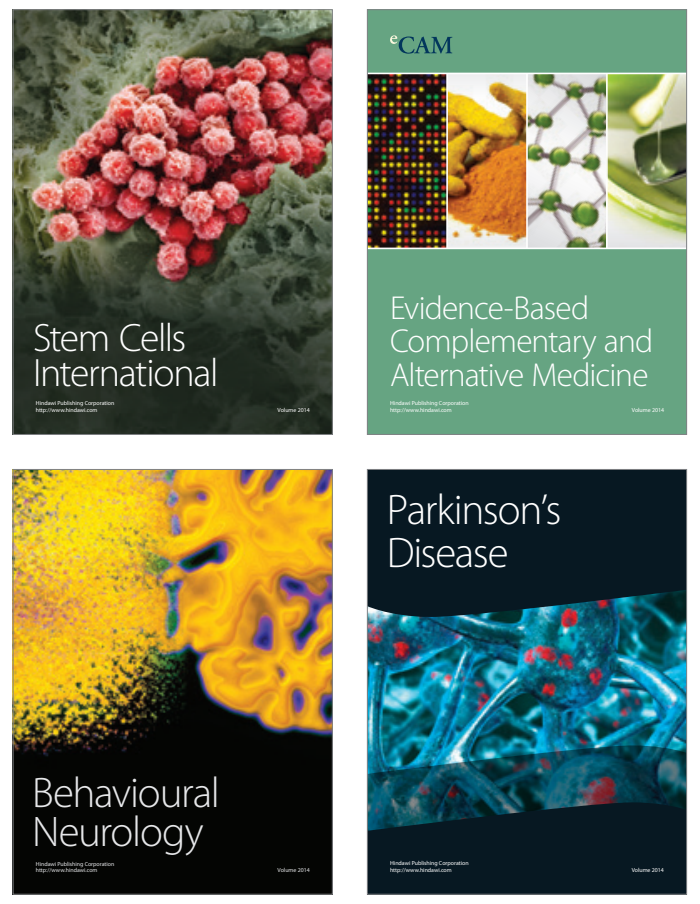
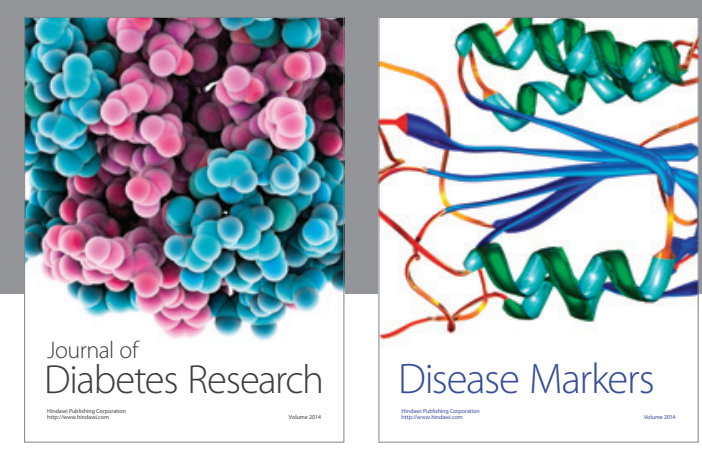

Disease Markers
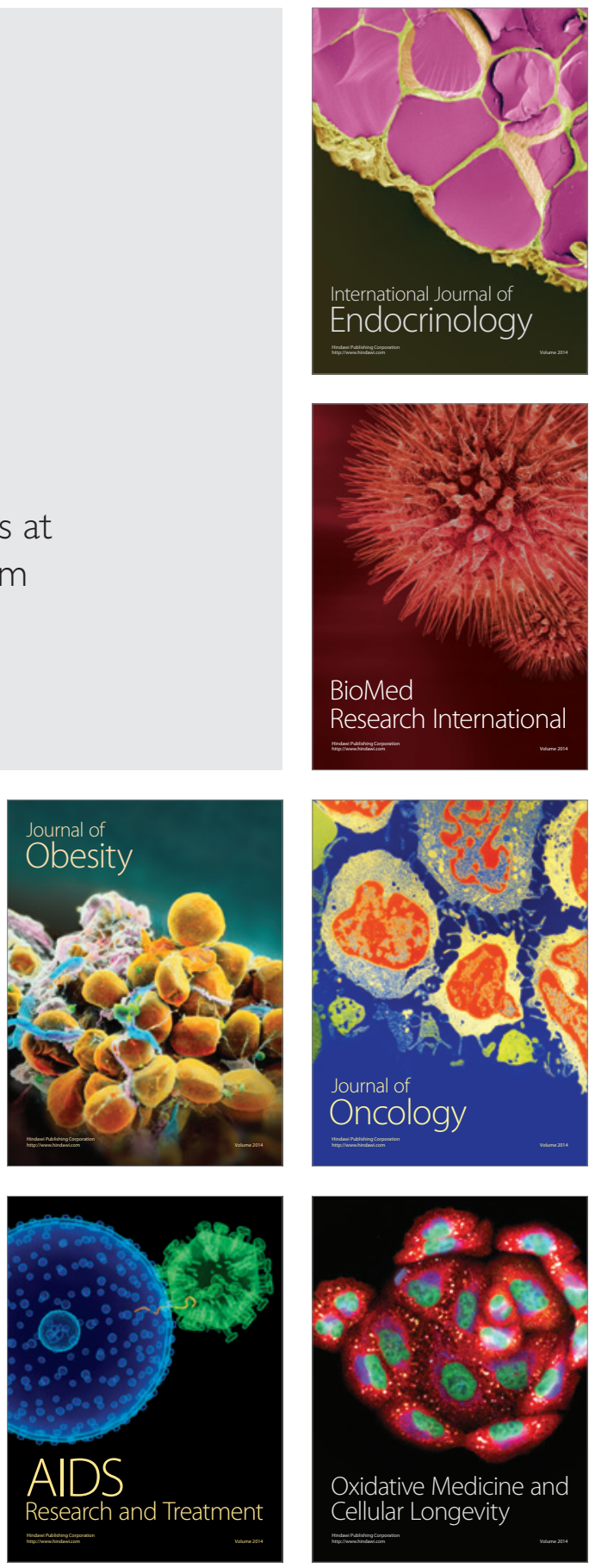